\title{
DIGITALCOMMONS
}

@WAYNESTATE -

Wayne State University

3-1-1997

\section{Ergodic Control of Reflected Diffusions with Jumps}

Jose-Luis Menaldi

Wayne State University, menaldi@wayne.edu

Maurice Robin

Centre Europen de Recherche Nucleare

\section{Recommended Citation}

Menaldi, J.L. \& Robin, M. Appl Math Optim (1997) 35: 117. doi: 10.1007/BF02683323

Available at: https://digitalcommons.wayne.edu/mathfrp/52

This Article is brought to you for free and open access by the Mathematics at DigitalCommons@WayneState. It has been accepted for inclusion in Mathematics Faculty Research Publications by an authorized administrator of DigitalCommons@WayneState. 


\title{
ERGODIC CONTROL OF REFLECTED DIFFUSIONS WITH JUMPS
}

\author{
Jose-Luis MenALDI* \\ Wayne State University \\ Department of Mathematics \\ Detroit, Michigan 48202, USA
}

\author{
MAURICE ROBIN ${ }^{\dagger}$ \\ CERN \\ $\mathrm{CH}-1211$ \\ Geneve 23, Suisse
}

July 27,2000

\begin{abstract}
We discuss ergodicity properties of a controlled jumps diffusion process reflected from the boundary of a bounded domain. The control parameters act on the drift term and on a first order type jump density. The controlled process is generated via a Girsanov change of probability, and a long run average criterion is to be optimized. By means of the Hamilton-Jacobi-Bellman equation, an optimal stationary feedback is constructed.
\end{abstract}

Key words and phrases: Reflected jump diffusion, ergodic optimal control, dynamic programming principle, Hamilton-Jacobi-Bellman equation, Green function, Girsanov transformation, Doeblin condition, discounted control problem.

1991 AMS Subject Classification. Primary: 49A60, 60J75, 36J60. Secondary: 49L20, 93D05, 93E20.

*e-mail: jlm@math.wayne.edu

${ }^{\dagger}$ e-mail: maurice_robin@macmail.cern.ch 


\section{Introduction}

We are interested in the ergodic properties of a jumps diffusion process reflected from the boundary of a bounded domain. Moreover, we intent to control the parameters of the drift and the jump terms in order to minimize an average cost per unit time over the infinity time interval. The main goal is to extend the results of $[23,25]$ to unbounded jumps measures

We will follow the model of Bensoussan [5, p. 162-171] for the diffusion processes and we add a jump term. It may seem a trivial extension but the difficulties are in proving the basic ergodicity properties of the jump diffusion processes.

In most of the cases, the existence and regularity of an unique invariant measure for each control is the starting point of any analysis (cf. Azema et al. [4], Kogan [19]). Sometimes this can be partially avoided by using Markov chains (cf. Borkar [8]), by means of more statistic assumptions on the processes (cf. Morimoto [26]), or by restraining the set of admissible feedbacks (cf. Bensoussan [5, p. 176]). More difficult settings are discussed in Gatarek and Stettner [17], Stettner [30], where several invariant measures may exist.

Usually the existence of an invariant density probability measure involves the verification of the so-called Doeblin condition, which requires a lower bound estimate of the transition density probability function associated with the given Markov process. A nice discussion on stationary distributions can be found in Ethier and Kurtz [11, pp. 238-253]. In our case, we have to deal with the transition density probability function of a jump diffusion, i.e. the Green function associated with an integro-differential equation (cf. Garroni and Menaldi $[12,13])$. Some results concerning the switching control can be found in Menaldi, Perthame and Robin [22].

The paper is organized as follows:

Section 1 gives the basic assumptions and properties of reflected diffusions with jumps.

Section 2 deals with the existence of a unique invariant measure for these processes.

Section 3 is devoted to study the Hamilton-Jacobi-Belmann equation of the ergodic control problem. 


\section{Basic Properties}

In this section we will describe the Markov-Feller process used to model the dynamic of the system.

\subsection{Reflected Diffusion with Jumps}

Consider an integro-differential operator of the form

$$
I_{0} \varphi(x)=\int_{\mathbb{R}_{\star}^{d}}[\varphi(x+z)-\varphi(x)-z \cdot \nabla \varphi(x)] M_{0}(s, d z)
$$

where the Levy kernel $M_{0}(x, d z)$ is a Radon measure on $\mathbb{R}_{\star}^{d}=\mathbb{R}^{d}-\{0\}$ for any fixed $x$, and satisfies

$$
\int_{|z| \leq 1}|z|^{2} M_{0}(x, d z)+\int_{|z|>1}|z| M_{0}(x, d z)<\infty, \forall x \in \mathbb{R}^{d}
$$

It is clear that this operator is associated with a jumps process.

Similarly, let $L_{0}$ be a second order uniformly elliptic operator associated with a diffusion process, i.e.

$$
L_{0}=\sum_{i, j=1}^{d} a_{i j}(x) \partial_{i j}
$$

where, the coefficients $\left(a_{i j}\right)$ are bounded and Hölder continuous, i.e. for some $c_{0}, M>0$, and $0<\alpha<1$

$$
\left\{\begin{array}{l}
c_{0}|\xi|^{2} \leq \sum_{i, j=1}^{d} a_{i j}(x) \xi_{i} \xi_{j} \leq c_{0}^{-1}|\xi|^{2}, \quad \forall x, \xi \in \mathbb{R}^{d} \\
\left|a_{i j}(x)-a_{i j}\left(x^{\prime}\right)\right| \leq M\left|x-x^{\prime}\right|^{\alpha}, \quad \forall x, x^{\prime} \in \mathbb{R}^{d}
\end{array}\right.
$$

and $a_{i j}=a_{j i}$, for $i, j=1, \ldots, d$.

The Levy kernel $M_{0}(x, d z)$ is assumed to have a particular structure, namely

$$
M_{0}(x, A)=\int_{\{\zeta: j(x, \zeta) \in A\}} m_{0}(x, \zeta) \pi(d \zeta)
$$

where $\pi(\cdot)$ is a $\sigma$-finite measure on the measurable space $(f, \mathcal{F})$, the functions $j(x, \zeta)$ and $m(x, \zeta)$ are measurable for $(x, \zeta)$ in $\mathbb{R}^{d} \times F$, and there exist a measurable and positive function $j(\zeta)$ and constants $C_{0}>0,1 \leq \gamma<2$ such that for every $x, \zeta$ we have

$$
\left\{\begin{array}{l}
|j(x, \zeta)| \leq j_{0}(\zeta), \quad 0 \leq m_{0}(x, \zeta) \leq 1 \\
\int_{F}\left[j_{0}(\zeta)\right]^{p}\left(1+j_{0}(\zeta)\right)^{-1} \pi(d \zeta) \leq C_{0}, \quad \forall p \in[\gamma, 2],
\end{array}\right.
$$


the function $j(x, \zeta)$ is continuously differentiable in $x$ for any fixed $\zeta$ and there exists a constant $c_{0}>0$ such that for any $(x, \zeta)$ we have

$$
c_{0} \leq \operatorname{det}(\mathbf{1}+\theta \nabla j(x, \zeta)) \leq c_{0}^{-1}, \quad \forall \theta \in[0,1]
$$

where 1 denotes the identity matrix in $\mathbb{R}^{d}, \nabla$ is the gradient operator is $x$, and $\operatorname{det}(\cdot)$ denotes the determinant of a matrix.

Notice that assumption (1.6) [resp. (1.7)] gives "control" of the $L^{\infty}\left(\mathbb{R}^{d}\right)\left[\operatorname{resp.} L^{1}\left(\mathbb{R}^{d}\right)\right]$ norm of the integro-differential operator $I_{0}$.

In order to develop the analysis on a bounded and smooth region $\mathcal{O}$ of $\mathbb{R}^{d}$, we give a first order differential operator defined on the boundary $\partial \mathcal{O}$, namely

$$
B=\sum_{i=1}^{d} b_{i}(x) \partial_{i},
$$

where the coefficients are non-tangential and continuously differentiable with a Hölder continuous derivative, i.e., for some constants $c_{0}>0,0<\alpha<1$, we have

$$
\left\{\begin{array}{l}
\sum_{i=1}^{d} b_{i}(x) n_{i}(x) \geq c_{0}, \quad \forall x \in \partial \mathcal{O}, \\
b_{i} \in C^{1+\alpha}(\partial \mathcal{O})
\end{array}\right.
$$

where $n=\left(n_{1}(x), \ldots, n_{d}(x)\right)$ denotes the unit outward normal to $\mathcal{O}$ at the point $x$ of the boundary $\partial \mathcal{O}$. Moreover, we may assume that $b_{i}$ are defined in the whole space $\mathbb{R}^{d}$, where $b_{i}$ and $\nabla b_{i}$ are bounded.

Now we need to "localize" the nonlocal operator $I_{0}$. For the sake of simplicity, we give the assumptions only for the case where $\mathcal{O}$ is convex, namely

$$
x+\theta j(x, \zeta) \in \overline{\mathcal{O}}, \quad \forall x \in \overline{\mathcal{O}}, \quad \forall \zeta \in F, \quad \forall \theta \in[0,1]
$$

This assumption (1.10) makes sense even for non convex domains, but it is more restrictive in that case.

Depending on the assumptions on the coefficients of the operators $L_{0}, I_{0}, B$ and on the domain $\mathcal{O}$, we can construct the corresponding Markov-Feller process. In the whole space, i.e. $\mathcal{O}=\mathbb{R}^{d}$, classic results are well know (e.g. Bensoussan and Lions [6], Gikhman and Skorokhod [18], and references therein), but in a bounded region with oblique derivative (i.e. $B$ different of the co-normal operator of $L_{0}$ ) only a few results are available. For 
instance we refer to Anulova [2, 3], Bony et al. [8] and Chaleyat-Maurel et al. [10]. However, some regularity on the coefficients $j(x, \zeta)$ and $m_{0}(x, \zeta)$ is needed, namely

$$
\left\{\begin{array}{l}
\left|m(x, \zeta)-m\left(x^{\prime} \zeta\right)\right| \leq M\left|x-x^{\prime}\right|^{\alpha}, \quad \forall x, x^{\prime} \in \mathbb{R}^{d} \\
\left|j(x, \zeta)-j\left(x^{\prime}, \zeta\right)\right| \leq j_{0}(\zeta)\left|x-x^{\prime}\right|^{\alpha}, \quad \forall x, x^{\prime} \in \mathbb{R}^{d}
\end{array}\right.
$$

for some constant $M>0$ and the same function $j(\zeta)$ as in assumption (1.6). Thus the integro-differential operator $I_{0}$ has the form

$$
I_{0} \varphi(x)=\int_{F}[\varphi(x+j(x, \zeta))-\varphi(x)-j(x, \zeta) \cdot \nabla \varphi(x)] m_{0}(x, \zeta) \pi(d \zeta) .
$$

To be more precise with the construction of the Markov-Feller process with infinitesimal generator $L_{0}+I_{0}$, we can mention that if $m_{0}(x, \zeta)=1$ and the coefficients $\left(a_{i i}\right)$ are Lipschitz continuous and $\left(b_{i}\right)$ and the domain $\mathcal{O}$ are of class $C^{3}$, then the penalization technique on the domain applied to the stochastic differential equation in the whole, space can be used (cf. [24]). Under the assumptions (1.4), (1.6), (1.7), (1.9), (1.10) and (1.11), we can construct the Markov-Feller process as a consequence of the specific estimates on the Green function (cf. Garroni and Menaldi $[12,13]$ ).

Since our goal is to study the ergodic control problem and the key starting point is to have good estimates on the transition probability function (i.e., the Green function), we will recall some results proved in the reference above.

Define the seminorms $C(\varphi, k)$ and $K(\varphi, k)$ of order $k>0$, for a kernel function $\varphi(x, t, y)$ as follows:

$$
C(\varphi, k)=\inf \left\{C \geq 0:|\varphi(x, t, y)| \leq C(t \wedge 1)^{-1+\frac{k-d}{2}}, \forall x, t, y\right\}
$$

and

$$
\left\{\begin{aligned}
K(\varphi, k)=\inf \{K \geq & 0: \int_{\mathcal{O}}[(\varphi(x, t, z)|+| \varphi(z, t, y) \mid] d z \leq \\
& \left.\leq C(t \wedge 1)^{-1+\frac{k}{2}}, \quad \forall x, t, y\right\}
\end{aligned}\right.
$$

where $\wedge$ denotes the minimum between two numbers. Denote by $\mathcal{G}_{k}$ the Banach space of kernels $\varphi(x, t, y),(x, t, y)$ in $\overline{\mathcal{O}} \times(0, \infty) \times \overline{\mathcal{O}}$ such that $C(\varphi, k)$ and $K(\varphi, k)$ are finite.

On the other hand, it is known that under the assumption (1.4) and (1.9) there exists a Green function $G_{0}(x, t, y)$ corresponding to the operator $L_{0}$ and $B$, which satisfies all the classic "heat-kernel" type estimates (e.g. Garroni and Solonnikov [15]). 
Theorem 1.1 Let the assumptions (1.4), (1.6) $[0<\gamma<2]$, (1.7), (1.9), (1.10), (1.11) and

$$
\mathcal{O} \text { is a bounded domain with a } C^{2+\alpha} \text { boundary } \partial \mathcal{O} \text {, }
$$

hold. Then there exists a diffusion process with jumps in $\overline{\mathcal{O}}$, with oblique boundary reflection, whose transition density probability function $G(x, t, y)$ can be represented as

$$
G=G_{0}+G_{0} \bullet Q
$$

where $Q$ is the unique kernel solution in the Green space $\mathcal{G}_{2-\gamma}$ of the Volterra equation

$$
Q=Q_{0}+Q_{0} \bullet Q, \quad Q_{0}=I_{0} G_{0}
$$

Comments. First, the symbol • means the "kernel convolution",i.e.

$$
\left(Q_{0} \bullet Q\right)(x, t, y)=\int_{0}^{t} d s \int_{\mathcal{O}} Q_{0}(x, t-s, z) Q(z, s, y) d z .
$$

The fact that we call $G$ a transition density probability function of a reflected diffusion with jumps is because its infinitesimal generator is $L_{0}+I_{0}$, we have precisely the following properties:

(i) for any smooth function $f$ with a compact support in $\mathcal{O} \times(0, \infty)$, the domain potential

$$
u(x, t)=\int_{0}^{t} d s \int_{\mathcal{O}} G(x, t-s, y) f(y, s) d y \doteq(G \bullet f)(x, t)
$$

is a solution in $W_{p, \text { loc }}(\mathcal{O} \times] 0, \infty[)$, for any $p$ in $(1, \infty)$, of the equation

$$
\left.\partial_{t} u-L_{0} u-I_{0} u=f, \text { a.e. in } \mathcal{O} \times\right] 0, \infty[
$$

(ii) for any continuous function $h$ with a compact support in $\mathcal{O}$, the potential

$$
w(x, t)=\int_{\mathcal{O}} G(x, t, y) h(y) d y
$$

is continuous in $\mathcal{O} \times[0, \infty)$ and satisfies the limit condition

$$
\lim _{t \rightarrow \infty} w(x, t)=h(x), \quad \forall x \in \mathcal{O} ;
$$

\footnotetext{
${ }^{1}$ the assumption (1.11) is not actually needed, it is assumed to hold for the sake of simplicity.
} 
(iii) for any smooth function $f$ with a compact support in $\mathcal{O} \times(0, \infty)$, the domain potential $u(x, t)$ is smooth up to the boundary [i.e., belongs to $W_{p}^{2,1}(\mathcal{O} \times] 0, T[)$, for any $T>0$ ] and satisfies the boundary condition

$$
B u=0 \text {, a.e. in } \partial \mathcal{O} \times] 0, \infty[\text {, }
$$

as a trace in the corresponding Sobolev space.

Actually, because we can always suppose $\gamma<2-\alpha$ for $\alpha$ sufficiently small, the domain potential $u(x, t)$ given by $(1.19)$ is smooth, i.e., belongs to the Hölder space $C^{2+\alpha, 1+\frac{\alpha}{2}}(\overline{\mathcal{O}} \times$ $[0, T]), \forall T>0$. Since we are planning to add first order terms to both operators $L_{0}$ and $I_{0}$, we choose the above formulation in Sobolev spaces. These first order terms will be only bounded and measurable instead of Hölder continuous.

Notice that since the function $f$ in (1.19) has a compact support in $\mathcal{O} \times(0, \infty)$, the compatibility condition

$$
B \varphi=\psi \text { in } \partial \mathcal{O} \times\{0\}
$$

is clearly satisfied. Here $\varphi$ is the initial data for $t=0$ (i.e, $\varphi=0$ ) and $\psi$ is the boundary data on $\partial \mathcal{O}$ (i.e, $\psi=0)$.

Sketch of the Proof. We refer to Theorem VIII.3.3 in Garroni and Menaldi [13] to establish the result is $\overline{\mathcal{O}} \times[0, T]$, for any $T>0$, and then we use the argument of Proposition 3.1 in Garroni and Menaldi [12] to conclude. We will outline briefly the main steps of the proof.

It is first proven the following "kernel convolution" estimates

$$
\begin{aligned}
& \left\{\begin{aligned}
& C(\psi \bullet \varphi, k+r) \leq 2^{2+d}\left(r^{-1}+k^{-1}\right)[C(\psi, r) K(\varphi, k)+ \\
&+K(\psi, r) C(\varphi, k)], \quad 0<k \leq d, \\
& C(\psi \bullet \varphi, k+r) \leq \beta\left(\frac{r}{2}, \frac{k-d}{2}\right) K(\psi, r) C(\varphi, k), \quad k>d,
\end{aligned}\right. \\
& K(\psi \bullet \varphi, k+r) \leq \beta\left(\frac{r}{2}, \frac{k}{2}\right) K(\psi, r) K(\varphi, k),
\end{aligned}
$$

where $\beta(\cdot, \cdot)$ denotes the $\beta$-function. 
Next, a detailed calculation proves an estimate on the integro-differential operator $I_{0}$, with $1 \leq \gamma<2$, namely

$$
\left\{\begin{array}{l}
C\left(I_{0} \varphi, k-\gamma\right) \leq C_{0}\left[2 C(\nabla \varphi, k-1)+C\left(\nabla^{2} \varphi, k-2\right)\right] \\
K\left(I_{0} \varphi, k=\gamma\right) \leq c_{0} C_{0}\left[2 K(\nabla \varphi, k-1)++K\left(\nabla^{2} \varphi, k-2\right)\right]
\end{array}\right.
$$

where $c_{0}$ and $C_{0}$ are the constants in assumptions (1.6) and (1.7).

By means of the above estimates one can proves that the Volterra equation (1.17) has one and only one solution given by the series

$$
Q=\sum_{n=0}^{\infty} Q_{n}, \quad Q_{n}=Q_{0} \bullet Q_{n-1}
$$

which is convergent in the Green space $\mathcal{G}_{2-\gamma}$ on $\overline{\mathcal{O}} \times(0, T]$, for every $T>0$.

Thus, defining $G$ by (1.16), it is proven that $G$ satisfies all the required properties of a transition density probability function, in particular, the conditions (i), (ii), (iii) above and the semigroup property are satisfied. For instance, to prove (i) and (iii) above, we use the properties of the initial Green function $G_{0}$ and the Volterra equation (1.17). Property (ii) above follows from the estimate (1.25) applied to $\psi=G_{0}, r=2$ and $\varphi=Q, k=2-\gamma$. This shows that

$$
\left|\int_{\mathcal{O}}\left(G_{0} \bullet Q\right)(x, t, y) h(y) d y\right| \leq C t^{\frac{2-\gamma}{2}}
$$

for any $0<t \leq 1$ and some constant $C$. The semigroup property is inherited from $G_{0}$ as a consequence of the uniqueness of the Volterra equation (1.17).

Actually, by taking $0<\alpha<1$ sufficiently small so that $\gamma<2-\alpha$, the kernel solution to the Volterra equation (1.17) belongs to the Green space $\mathcal{G}_{2-\gamma}^{\alpha}$ (which includes Hölder type seminorms, see the mentioned references for more detail) and the Green function $G_{0} \bullet Q$ belongs to the Green space denoted by $\mathcal{G}_{4-\gamma}^{2+\alpha, 1+\frac{\alpha}{2}}$.

Let us discuss in more detail the positivity of the Green function in order to obtain a transition density probability function and then to be able to construct an associated Markov-Feller process. (e.g. Theorem III.2.7 in Ethier and Kurtz [11]). It is clear that a weak version of the maximum principle will be necessary at this point, i.e. the statement either

$$
G(x, t, y) \geq 0, \quad \forall x, t, y
$$




$$
f \geq 0 \text { implies } G f \geq 0
$$

for any smooth function $f$ with a compact support in $\mathcal{O}_{\times}(0, \infty)$.

Since, we really want to prove the strict positivity of the Green function $G$, we will recall a version of the (classic) maximum principle for integro-differential operator as proved in Garroni and Menaldi [14].

Theorem 1.2 (Strong Maximum Principle) Let us assume (1.4), (1.6), (1.7), (1.9) and (1.10) [(1.11) is not necessary] hold true. Suppose that a function $u(x, t)$ satisfies

$$
\left\{\begin{array}{l}
u \in C^{2,1}(\mathcal{O} \times(0, T]) \cap C^{1,0}(\overline{\mathcal{O}} \times(0, T]) \cap C^{0}(\overline{\mathcal{O}} \times[0, T]), \\
\partial_{t} u-L_{0} u-I_{0} u \leq 0 \text { in } \mathcal{\mathcal { O }} \times(0, T] \\
B u \leq 0 \text { in } \partial \mathcal{O} \times(0, T]
\end{array}\right.
$$

If $u$ is not a constant function then the maximum value of $u$ on $\overline{\mathcal{O}} \times(0, T]$ must be attained on $\overline{\mathcal{O}} \times\{0\}$.

It is clear that a simple application of the strong maximum principle (actually, a weak version is sufficient) provides (1.31), and by continuity we obtain (1.30). However, to show the strict positivity of the Green function, i.e., for any $\delta>0$ there exists a positive constant $c=c(\delta)>0$ such that

$$
G(x, t, y) \geq c, \quad \forall x, y \in \overline{\mathcal{O}}, \quad \forall t \geq \delta
$$

the argument is little more complicate. First, by means of the semigroup property

$$
G(x, t+s, y)=\int_{\mathcal{O}} G(x, t, z) G(z, s, y) d z
$$

and the identity

$$
\int_{\mathcal{O}} G(x, t, z) d z=1
$$

we reduce the inequality (1.33) to the case where $t=\delta>0$. Now, let us prove that $G(x, \delta, y)>0$ for any $x, y$ in $\mathcal{O}$. Indeed, by contradiction, if $G$ vanishes at point $P_{0}=$ $\left(x_{0}, \delta, y_{0}\right)$ then $P_{0}$ is a point where $G$ attains its minimum value (since we know that $G \geq 0$ ). 
Thus the strong maximum principle applied to the function $u(x, t)=-G\left(x, t+\frac{\delta}{2}, y_{0}\right)$ gives a contradiction. Next, by means of the continuity in $x$ and $y$ we deduce assertion (1.33).

Notice that in order to use weak maximum principle, we used the fact that the domain potential $u(x, t)$ given by $(1.19)$ is smooth [i.e., it satisfies the regularity assumptions in (1.32)]. However, to be able to deduce the strict positivity of the Green function $G$, we need to know that $G(x, t, y)$ is smooth in $x, t$ [only $t>0$ ] for any fixed $y$, and that $G(x, t, y)$ is continuous in $(x, y)$ for any $t>0$ fixed. These two conditions are a direct consequence of the representation (1.16) in Theorem 1.1 and the fact that $G_{0} \bullet Q$ belongs to the Green space $\mathcal{G}_{4-\gamma}^{2+\alpha, 1+\frac{\alpha}{2}}$ of a Hölder type, provided that (1.11) is satisfied.

Notice that a maximum principle for integro-differential operator (of the type $L_{0}+I_{0}$ ) valid on the Sobolev space $W_{p}^{2,1}$ (similar to that of Bony [7] and Krylov [20] for differential operators) has not yet been established. However, if we add some more assumptions on the coefficient of $L_{0}$ and $I_{0}$ (such as Lipschitz continuity of $a_{i j}$ ) then the operator $L_{0}+I_{0}$ are in divergence form and a variational formulation is possible. Thus, the standard coercitivity assumptions (e.g. Bensoussan and Lions [6]) are sufficient to prove a weak version of the maximum principle.

\subsection{Measurable First Order Coefficients}

In order to accommodate the control parameters, we need to be able to construct a reflected diffusion with jumps where the first order coefficients are only bounded and measurable. To that purpose, we will use the so-called Girsanov's transformation.

Let $\Omega=D\left(\left[0, \infty\left[, \mathbb{R}^{d}\right)\right.\right.$ be the canonical space of right continuous functions $\omega$ from $[0, \infty[$ into $\mathbb{R}^{d}$ endowed with the Skorokhod topology. Denote by either $X_{t}$ or $X(t)$ the canonical process and by $F_{t}$ the filtration generated by $\left\{X_{s}: s \leq t\right.$. Now let $\left(\Omega, P, F_{t}, X(t), t \geq 0\right)$ be the (homogeneous) Markov-Feller process with transition density function $G(x, t, y)$ associated to the integro-differential operator $L_{0}+I_{0}$ and the boundary operator $B$ as described in the previous subsection, i.e. the density with respect to the Lebesgue measure of $P\{X(t) \in d y \mid X(s)=x\}$ is equal to $G(x, t-s, y)$. For the sake of simplicity, we will refer to $\left(P_{x}, X(t), t \geq 0\right)$ as the above Markov-Feller process, where $P_{x}$ denote the conditional probability with respect to $\{X(0)=x\}$. 
Hence, for any smooth function $\varphi(x)$ satisfying the boundary condition

$$
B \varphi=0 \text { on } \partial \mathcal{O}
$$

the process

$$
Y_{\varphi}(t)=\varphi(X(t))-\int_{0}^{t}\left(L_{0}+I_{0}\right) \varphi(X(s)) d s
$$

is a $P_{x}$-martingale. This follows immediately from the representation

$$
\left\{\begin{aligned}
E_{x}\{\varphi(X(t))\}= & \int_{\mathcal{O}} G(x, t, y) \varphi(y) d y+ \\
& +\int_{0}^{t} d s \int_{\mathcal{O}} G(x, t-s, y)\left(L_{0}+I_{0}\right) \varphi(y) d y
\end{aligned}\right.
$$

and the Markov property.

Hence, following the martingale approach (e.g. Theorems II.3.1 and II.3.2 in Bensoussan and Lions [6]) we deduce that there is a unique increasing (by coordinates), continuous and adapted process $(\xi(t), t \geq 0)$ with values in $\mathbb{R}^{d}$ such that

$$
\xi(t)=\int_{0}^{t} \chi(X(s) \in \mathcal{O}) d \xi(t)
$$

and

$$
Z_{\varphi}(t)=\varphi(X(t))+\int_{0}^{t} B \varphi(X(s)) d \xi(s)-\int_{0}^{t}\left(L_{0}+I_{0}\right) \varphi(X(s)) d s
$$

is a $P_{x}$-martingale for any smooth function $\varphi$.

Notice that in view of the analytic properties of the operator $L_{0}+I_{0}$ (with the boundary operator $B$ ) the martingale problem (1.37) identifies completely (i.e. the martingale problem has the uniqueness property) the Markov-Feller process $\left(P_{x}, X(t), t \geq 0\right)$.

It is also possible to express the process $X_{t}$ as follows:

$$
d X(t)=a^{1 / 2}(X(t)) d w(t)+\int_{\mathbb{R}_{\star}^{d}} z \mu_{X}(d t, d z)-b(X(t)) d \xi(t),
$$

where $(w(t), t \geq 0)$ is a standard Wiener process in $\mathbb{R}^{d},(\xi(t), t \geq 0)$ is the process satisfying (1.39), $a^{1 / 2}(x)$ is the positive square root of the matrix $\left(a_{i j}(x)\right)$ and $b(x)$ is the vector function $\left(b_{i}(x)\right)$. The process $\mu_{X}$ is the martingale measure associate with the process $(X(t), t \geq 0)$, i.e. if $\eta_{X}(t, A)$ denotes the integer random measure defined as the number of jumps of the process $X(t)$ on $(0, t)$ with values in $A \subset \mathbb{R}_{\star}^{d}$ then

$$
\mu_{X}(d t, A)+\pi_{X}(d t, A)=\eta_{X}(d t, A)
$$


where $\mu_{X}(t, A)$ is a square integrable (local) martingale quasi-left continuous and $\pi_{X}(t, A)$ is a predictable increasing process obtained via the Doob-Meyer decomposition. Moreover,

$$
\pi_{X}(d t, d z)=M_{0}(X(t-), d z) d t
$$

where $M_{0}(x, d z)$ is the Levy kernel used to defined the integro-differential operator $I_{0}$ given by (1.1).

Now, we are ready to introduce measurable first order coefficients via the Girsanov's transformation. Suppose that

$$
\left\{\begin{array}{l}
a_{j}(x), \ldots, a_{d}(x), \text { and } c(x, z) \text { are } \\
\text { measurable and bounded functions such that } \\
0 \leq c(x, z) \leq C_{0}(1 \wedge|z|), \quad \forall x \in \overline{\mathcal{O}}, \quad \forall z \in \mathbb{R}_{\star}^{d}
\end{array}\right.
$$

and let $(e(t), t \geq 0)$ the exponential martingale solution to the stochastic differential equation

$$
\left\{\begin{aligned}
d e(t) & =e(t)\left[r_{X}(t) d w(t)+\int_{\mathbb{R}_{\star}^{d}} \gamma_{X}(t, z) \mu_{X}(d t, d z)\right] \\
e(0) & =1
\end{aligned}\right.
$$

where

$$
\left\{\begin{array}{l}
r_{X}(t)=a^{-1 / 2}(X(t))\left(a_{1}(X(t)), \ldots, a_{d}(X(t))\right) \\
\gamma_{X}(t, z)=z c(X(t), z)
\end{array}\right.
$$

i.e.,

$$
\left\{\begin{aligned}
e(t)= & \exp \left\{\int_{0}^{t} r_{X}(s) d w(s)+\int_{0}^{t} \int_{\mathbb{R}_{\star}^{d}} \gamma_{X}(s, z) \mu_{X}(d s, d z)-\right. \\
& \left.-\int_{0}^{t}\left|r_{X}(s)\right|^{2} d s-\int_{0}^{t} \int_{\mathbb{R}^{d}}\left[\gamma_{X}(s, z)-\ln \left(1+\gamma_{X}(s, z)\right)\right] \pi_{X}(d s, d z)\right\} .
\end{aligned}\right.
$$

If we denote by

$$
L=L_{0}+\sum_{i=1}^{d} a_{i}(x) \partial_{i}
$$

and

$$
I \varphi(x)=I_{0} \varphi(x)+\int_{\mathbb{R}_{\star}^{d}}[\varphi(x+z)-\varphi(x)] c(x, z) M_{0}(x, d z),
$$

then, by means of Itô's formula we prove that for any smooth function $\varphi$, the process

$$
Z_{\varphi}=\varphi(X(t))+\int_{0}^{t} B \varphi(X(s)) d \xi(s)-\int_{0}^{t}(L+I) \varphi(X(s)) d s
$$


is a $P_{x}^{e}$-martingale, where the new probability measure is defined as

$$
d P_{x}^{e}=e(t) d P_{x} \quad \text { on } \quad F_{t}
$$

Thus, $\left(P_{x}^{e}, X(t), t \geq 0\right)$ is a Markov-Feller process on the canonical space $D([0, \infty[, \overline{\mathcal{O}})$ whose infinitesimal generator is an extension of the integro-differential operator $L+I$ [given by (1.48), (1.49)] defined for $C^{2}$ function satisfying the boundary condition (1.36).

Notice that the probability measures $P_{x}$ and $P_{x}^{e}$ are absolutely continuous one with respect to each other. Moreover, by expressing the second part of the integro-differential operator $I$ in $(1.49)$ as

$$
\int_{A} c(x, z) M_{0}(x, d z)=\int_{\{\xi: j(x, \xi) \in A\}} m_{0}(x, \xi) \pi(d \xi)
$$

where $\pi(\cdot), j(\cdot, \cdot)$ and $m_{0}(\cdot, \cdot)$ are as in (1.5), (1.6), (1.7), and $m(x, \xi)$ is a measurable function for $(x, \zeta)$ in $\overline{\mathcal{O}} \times F$ such that

$$
0 \leq m(x, \zeta) \leq C_{0}, \quad \forall x, \zeta
$$

we obtain a transition density function $G(x, t, \zeta)$ satisfying the same condition (with obvious changes) mentioned in Theorem 1.1. The difference is that now the Green function may not be continuous in the variable $y$, in particular the conditions (i), (ii) and (iii) hold in Sobolev spaces (as stated, and not in Hölder spaces). In this case, only a weak version of the maximum principle is known [i.e. (1.30) follows by regularization]. The strict positivity of the Green function is yet to be proved.

\section{Invariant Measure}

First in this section we will formulate the ergodic control problem and then we will discuss the associated invariant measure. 


\subsection{Ergodic Problem}

Let $f(x, v), g(x, v)=\left(g_{1}(x, v), \ldots, g_{d}(x, v)\right)$, and $c(x, v, z)$ functions defined for $(x, v)$ in $\overline{\mathcal{O}} \times V, z$ in $\mathbb{R}_{\star}^{d}$ such that

$$
\left\{\begin{array}{l}
f, g_{i}, c \text { are bounded and measurable, } \\
\text { and continuous in the control variable } v \\
0 \leq c(x, v, z) \leq C_{0}(1 \wedge|z|), \quad \forall x, v, z
\end{array}\right.
$$

where $C_{0}$ is a constant and $V$ is a compact metric space.

We consider the Markov-Feller process $\left(P_{x}, X(t), t \geq 0\right)$ defined on the canonical space $D\left(\left[0, \infty[, \overline{\mathcal{O}})\right.\right.$ described in Section 1 , corresponding to the integro-differential operator $L_{0}+$ $I_{0}$ with boundary differential operator $B$, given by (1.1), (1.3) and (1.8).

An admissible control is a stochastic process $(v(t), t \geq 0)$ with values in $V$, adapted to the filtration $F_{t}$. For any admissible control $(v(t), t \geq 0)$ we can use the Girsanov's transformation (1.47), (1.51) to define an exponential martingale $e_{v}(t)$ and a new probability measure denoted by $P_{x}^{v}$ such that $\left(P_{x}^{v}, X(t), t \geq 0\right)$ represents the state of the system. Notice that in this case, $e_{v}(t)$ is given by (1.47) with

$$
\left\{\begin{array}{l}
r_{X}(t)=a^{-1 / 2}(X(t)) g(X(t), v(t)) \\
\gamma_{X}(t, z)=z c(X(t), v(t), z)
\end{array}\right.
$$

A cost is associated with the controlled system $\left(P_{x}^{v}, X(t), t \geq 0\right)$ by

$$
J_{x}(v)=\lim _{T \rightarrow \infty} E_{x}^{v}\left\{\frac{1}{T} \int_{0}^{T} f(X(t), v(t)) d t\right\} .
$$

Our purpose is to give a characterization of the optimal cost

$$
\lambda=\inf \left\{J_{x}(v): v(\cdot)\right\}
$$

and to construct an optimal control $\hat{v}(t)$.

It is useful to remark that we expect to obtain an optimal Markovian control, i.e.

$$
\hat{v}(t)=\hat{v}(X(t)), \quad \forall t \geq 0
$$

for some feedback function $\hat{v}(x)$ and to prove that the optimal cost $\lambda$ is constant, i.e., independent of the initial condition $X(0)=x$. 
For a given feedback $v=v(x)$, the controlled state of the system $\left(P_{x}^{v}, X(t), t \geq 0\right)$ is a Markov-Feller process with infinitesimal generator of the form (1.48), with

$$
\begin{cases}\left(a_{1}(x), \ldots, a_{d}(x)\right) & =g(x, v(x)), \\ c(x, z) & =c(x, v(x), z) .\end{cases}
$$

Moreover, this Markov-Feller process $\left(P_{x}^{v}, X(t), t \geq 0\right)$ has a transition probability density function denoted by $G_{v}(x, t, y)$, which enjoys the properties described in Theorem 1.1, except that now $Q_{0}=\left(L+I-L_{0}\right) G_{0}$.

\subsection{Doeblin Condition}

As in Bensoussan [5], a key point in the study of ergodic control problems is to establish the so-called Doeblin condition. This property is usually a consequence of the strong maximum principle or/and the strict positivity of the Green functions. In our setting, those properties are true for the uncontrolled (or smooth) process $\left(P_{x}, X(t), t \geq 0\right)$, but do not hold (a priori) for the controlled process $\left(P_{x}^{v}, X(t), t \geq 0\right)$.

Denote by $G(x, t, y)$ and $G_{v}(x, t, y)$ the transition probability density functions corresponding to the "uncontrolled" process $\left(P_{x}, X(t), t \geq 0\right)$ and the controlled process $\left(P_{x}^{v}, X(t), t \geq 0\right)$ for a given Borel measurable feedback control $v=v(x)$.

The associated semigroups are

$$
\Phi(t) \varphi=\int_{\mathcal{O}} G(\cdot, t, y) \varphi(y) d y
$$

and

$$
\Phi_{v}(t) \varphi=\int_{\mathcal{O}} G_{v}(\cdot, t, y) \varphi(y) d y
$$

with the infinitesimal generator given by $L_{0}+I_{0}$ [cf. (1.1) and (1.3)] and $L_{v}+I_{v}$ where

$$
L_{v}=L_{0}+\sum_{i=1}^{d} g_{i}(x, v(x)) \partial_{i}
$$

and

$$
I_{v} \varphi(x)=I_{0} \varphi(x)+\int_{\mathbb{R}_{\star}^{d}}[\varphi(x+z)-\varphi(x)] c(x, v(x), z) M_{0}(x, d z) .
$$


As a consequence of the regularity of the Green function given in Theorem 1.1, we deduce that the strong Feller property is satisfied, i.e.,

$$
\left\{\begin{array}{l}
\text { for any } T, \varepsilon>0 \text { there exists } \delta=\delta(\varepsilon, T) \text { such that } \\
\text { for every } x, x^{\prime} \text { in } \overline{\mathcal{O}},\left|x-x^{\prime}\right|<\delta \text {, and } t \text { in }\left[T^{-1}, T\right] \text { one has } \\
\left|E_{x}^{v}\{\varphi(X(t))\}-E_{x^{\prime}}^{v}\{\varphi(X(t))\}\right| \leq \varepsilon\|\varphi\|, \\
\text { for any measurable and bounded function } \varphi,
\end{array}\right.
$$

where $\|\cdot\|$ denote the supremum norm on $\overline{\mathcal{O}}$ and $E_{x}^{v}$ is the conditional expectation with respect to the probability measure $P_{x}^{v}$ as before.

Theorem 2.1 (Doeblin condition) Let $v=r(x)$ be a Borel measurable feedback, and suppose that (1.4), (1.6), (1.7), (1.9), (1.10), (1.11), (1.15) and (2.1) hold. Then the Doeblin condition is satisfied for the controlled process $\left(P_{x}^{v}, X(t), t \geq 0\right)$, i.e.

$$
\left\{\begin{array}{l}
\text { there exists a constant } \delta>0 \text { such that } \\
\Phi_{v}(1) \varphi(x)-\Phi_{v}(1) \varphi\left(x^{\prime}\right) \leq 1-\delta \\
\text { for any } x, x^{\prime} \text { in } \overline{\mathcal{O}} \text { and any measurable } \\
\text { function } \varphi \text { satisfying } 0 \leq \varphi \leq 1 .
\end{array}\right.
$$

Proof. The argument is simple, first we check that (2.12) holds for the initial process $(P, X(t), t \geq 0)$ and then we prove that the property remains valid after a Girsanov change of probability measures.

Indeed, first for the uncontrolled process $\left(P_{x}, X(t), t \geq 0\right)$ we have

$$
\left\{\begin{aligned}
\Phi(1) \varphi(x)-\Phi(1) \varphi\left(x^{\prime}\right)= & 1-\left\{\int_{\mathcal{O}} G(x, 1, y)[1-\varphi(y)] d y+\right. \\
& \left.+\int_{\mathcal{O}} G\left(x^{\prime}, 1, y\right) \varphi(y) d y\right\} \leq \\
\leq & 1-c|\mathcal{O}|
\end{aligned}\right.
$$

where $|\mathcal{O}|$ is the measure of the set $\mathcal{O}$ and $c$ is the constant minorant the Green function as given by (1.33). Thus (2.12) holds for the uncontrolled process.

Now, assume that (2.12) does not hold for the controlled process $\left(P_{x}^{v}, X(t), t \geq 0\right)$. Then there exist sequences $x_{k}, x_{k}^{\prime}, \varphi_{k}, k=1,2, \ldots$, such that

$$
\begin{aligned}
& \Phi_{v}(1) \varphi_{k}\left(x_{k}\right) \rightarrow 1, \\
& \Phi_{v}(1) \varphi_{k}\left(x_{k}^{\prime}\right) \rightarrow 0 .
\end{aligned}
$$


Because $x_{k}, x_{k}^{\prime}$ belong to $\overline{\mathcal{O}}$ and $0 \leq \varphi_{k} \leq 1$, we can extract subsequences such that

$$
\begin{aligned}
& x_{k} \rightarrow x, \quad x_{k}^{\prime} \rightarrow x^{\prime} \text { and } \\
& \varphi_{k} \rightarrow \varphi \text { weakly } \star \text { in } L^{\infty}(\mathcal{O}) .
\end{aligned}
$$

Therefore, in view of the inequalities

$$
\begin{aligned}
1-E_{x}^{v}\left\{\varphi_{k}(X(1))\right\} \leq & \left|E_{x}^{v}\left\{\varphi_{k}(X(1))\right\}-E_{x_{k}}^{v}\left\{\varphi_{k}(X(1))\right\}\right|+ \\
& +\left[1-E_{x_{k}}^{v}\left\{\varphi_{k}(X(1))\right\}\right] \\
E_{x^{\prime}}^{v}\left\{\varphi_{k}(X(1))\right\} \leq & \left|E_{x^{\prime}}^{v}\left\{\varphi_{k}(X(1))\right\}-E_{x_{k}^{\prime}}^{v}\left\{\varphi_{k}(X(1))\right\}\right|+ \\
+ & E_{x_{k}^{\prime}}^{v}\left\{\varphi_{k}(X(1))\right\}
\end{aligned}
$$

and by means of the strong Feller property (2.11) we deduce

$$
E_{x}^{v} \varphi(X(1))=1 \quad \text { and } \quad E_{x^{\prime}}^{v} \varphi(X(1))=0 .
$$

Since the probability measures $P_{x}$ and $P_{x}^{v}$ are absolutely continuous one with respect to each other, we obtain

$$
E_{x}\{1-\varphi(X(1))\}=E_{x^{\prime}}\{\varphi(X(1))\}=0
$$

which contradict (2.13). Thus (2.12) has been established.

Once the Doeblin condition is satisfied, standard technique can be used to obtain a unique invariant measure (e.g. Bensoussan [5], Garroni and Menaldi [12], and Robin [28]). We summarize the results as follows.

Theorem 2.2 Under the assumptions of Theorem 2.1 there exists a unique bounded and measurable function $m=m_{v}(x)$ on $\mathcal{O}$ such that

$$
\left\{\begin{array}{l}
m(y)=\alpha \int_{0}^{\infty} e^{-\alpha t} d t \int_{\mathcal{O}} G_{v}(x, t, y) m(x) d x, \quad \forall \alpha>0 \\
\int_{\mathcal{O}} m(x) d x=1, \\
m(x) \geq 0, \text { a.e. in } \mathcal{O} .
\end{array}\right.
$$

Moreover, for every $t \geq 1$ and $h$ in $L^{1}(\mathcal{O})$ we have

$$
\left|\int_{\mathcal{O}} G_{v}(x, t, y) h(y) d y-\int_{\mathcal{O}} h(y) m(y) d y\right| \leq C e^{-\nu t}\|h\|_{L^{1}},
$$

for some constants $C, \nu>0$ independent of $t$ and $h$. Furthermore

$$
m(y)=\int_{\mathcal{O}} G_{v}(x, t, y) m(x) d x, \text { a.e. } y \text { in } \mathcal{O},
$$

for every $t>0$. 


\section{Hamilton-Jacobi-Bellman Equation}

First we give some useful results on the discounted HJB equation. Denote by $H(x, \varphi(x))$ the Hamiltonian

$$
\left\{\begin{aligned}
H(x, \varphi(x))=\inf \{ & \int_{\mathbb{R}_{\star}^{d}}[\varphi(x+z)-\varphi(x)] c(x, v, z) M_{0}(x, d z)+ \\
& +g(x, v) \cdot \nabla \varphi(x)+f(x, v): v \in V\}
\end{aligned}\right.
$$

for $\varphi$ in the Sobolev space $W^{1, p}(\mathcal{O}), d<p<\infty$ and $f$ in the Lebesgue space $L^{\infty}\left(V, L^{p}(\mathcal{O})\right)$. Actually, for the sake of simplicity, we are going to suppose that $f$ is bounded and measurable as in (2.1).

Theorem 3.1 Under the assumptions (1.4), (1.6), (1.7), (1.9), (1.10), (1.11) (1.15) and (2.1), the HJB equation

$$
\left\{\begin{array}{l}
L_{0} u_{\alpha}+I_{0} u_{\alpha}+H\left(\cdot, u_{\alpha}\right)=\alpha u_{\alpha} \text { in } \mathcal{O} \\
B u_{\alpha}=0 \text { on } \partial \mathcal{O}
\end{array}\right.
$$

has a unique solution $u_{\alpha}$ in $W^{2, p}(\mathcal{O})$, for every $\alpha>0$.

The proof of this theorem is very similar to the one in Bensoussan and Lions [5]. The key argument is based on the following estimates:

$$
\|I \varphi\|_{L^{p}(\mathcal{O})} \leq \varepsilon\left\|\nabla^{2} \varphi\right\|_{L^{p}(\mathcal{O})}+c(\varepsilon)\left[\|\varphi\|_{L^{p}(\mathcal{O})}+\|\nabla \varphi\|_{L^{p}(\mathcal{O})}\right]
$$

for any $\varepsilon>0$ and some constant $C(\varepsilon)$, where $I=I_{v}$ the integro-differential operator given by (2.10). We refer to Garroni et al. [16] for a more general results.

The above argument also applies to the linear equation

$$
\left\{\begin{array}{l}
L_{v} u_{\alpha}+I_{v} u_{\alpha}+h=\alpha u_{\alpha} \text { in } \mathcal{O} \\
B u_{\alpha}=0 \text { on } \partial \mathcal{O}
\end{array}\right.
$$

for any Borel measurable feedback $v=v(x), \alpha>0$ and the notation (2.9), (2.10). We have

Proposition 3.2 Let $v(x)$ be a Borel measurable and h a given function in $L^{p}(\mathcal{O})$. Assume the conditions (1.4), (1.6), (1.7), (1.9), (1.10), (1.15) and (2.1) [on g and c, only], then

\footnotetext{
${ }^{2}$ This condition (1.11) is not actually needed, it will be used in Theorem 3.5
} 
the linear equation (3.4) has a unique solution in $W^{2, p}(\mathcal{O})$. Moreover, it can be written under the following equivalent forms:

$$
\left\{\begin{array}{l}
u_{\alpha}=\int_{0}^{\infty} e^{-\alpha t} \Phi_{v}(t) h d t \\
u_{\alpha}(x)=\int_{0}^{\infty} e^{-\alpha t} d t \int_{\mathcal{O}} G_{v}(x, t, y) h(y) d y \\
u_{\alpha}(x)=E_{x}^{v}\left\{\int_{0}^{\infty} e^{-\alpha t} h(X(t)) d t\right\}
\end{array}\right.
$$

for any $\alpha>0$.

Let us now consider the undiscounted linear problem corresponding to (3.4), namely

$$
\left\{\begin{array}{l}
L_{v} w+I_{v} w+h=0 \text { in } \mathcal{O} \\
B w=0 \text { on } \partial \mathcal{O}
\end{array}\right.
$$

where $h$ has a zero mean, i.e.,

$$
\left\{\begin{array}{l}
h \in L^{p}(\mathcal{O}), \quad d<p<\infty \\
\bar{h} \doteq \int_{\mathcal{O}} h(x) m_{v}(x) d x=0,
\end{array}\right.
$$

where $m=m_{v}(x)$ is the invariant probability density associated with $v$ (cf. Theorem 2.2).

Proposition 3.3 Let $v(x)$ be a Borel measurable feedback. Assume that conditions (1.4), (1.6), (1.7), (1.9), (1.10), (1.11), (1.15), (2.1) [relative to $g$ and c only] and (3.7). Then problem (3.6) has a solution in $W^{2, p}(\mathcal{O})$, unique up to an additive constant.

Proof. We refer instant to Bensoussan [5], Garroni and Menaldi [12], and Robin [27]. The argument is as follows.

Let us denote by $w_{\alpha}$ the solution of discounted problem (3.4), $\alpha>0$, with $h$ satisfying (3.7). In view of Theorem 2.2 we have

$$
\left\|\Phi_{v}(t) h\right\|_{\infty} \leq C\|h\|_{1} e^{-\nu t}
$$

where $\|\cdot\|_{p}$ denotes the norm in $L^{p}(\mathcal{O}), 1 \leq p \leq \infty$. Thus, from the representation (3.5) we deduce

$$
\left\|w_{\alpha}\right\|_{\infty} \leq C, \quad \forall \alpha>0
$$

for some appropriate constant $C$ independent of $\alpha$. 
Therefore, the above estimate (3.9) combined with the $W^{2, p}$ estimates of the type Agmon, Douglas and Nirenberg[1] for the differential operator $L_{v}$ and the inequality (3.3) regarding the integro-differential operator $I_{v}$ yield

$$
\left\|w_{\alpha}\right\|_{W^{2, p}} \leq C, \quad \forall \alpha>0
$$

for another suitable constant $C$ independent of $\alpha$.

Thus, there exists a subsequence $w_{\alpha}$ converging to some function $w$ in $W^{2, p}$ weakly. Hence, we can let $\alpha$ go to zero in (3.4) and we obtain that $w$ satisfies (3.6).

In order to show that $w$ is unique up to an additive constant, it is enough to show that

$$
\begin{aligned}
& L_{v} u+I_{v} u=0 \text { in } \mathcal{O}, \\
& B u=0 \text { in } \partial \mathcal{O}
\end{aligned}
$$

implies that $u$ is a constant function. To that effect, let $u$ be a solution of the above equation. Then it is clear that

$$
\Phi_{v}(t) u=u \quad \forall t
$$

and since

$$
\Phi_{v}(t) u \rightarrow \int_{\mathcal{O}} u(x) m_{v}(d x)
$$

as $t \rightarrow \infty$, the desired result is proved.

Corollary 3.4 Let us consider the problem (3.4) without the assumption (3.7), its solution denoted by $u_{\alpha}(x)$. If we set

$$
w_{\alpha} \doteq u_{\alpha}-\bar{u}_{\alpha}=u_{\alpha}-\int_{\mathcal{O}} u_{\alpha}(x) m_{v}(x) d x
$$

then we have

$$
\left\{\begin{array}{l}
w_{\alpha} \rightarrow w \text { weakly in } W^{2, p}(\mathcal{O}), \\
\alpha u_{\alpha} \rightarrow \bar{h}
\end{array}\right.
$$

and $(w, \bar{h})$ satisfies

$$
\left\{\begin{array}{l}
L_{v} w+I_{v} w+h=\bar{h} \text { in } \mathcal{O} \\
B w=0 \quad \text { on } \partial \mathcal{O} \\
\bar{w}=0 . \square
\end{array}\right.
$$


We are now ready to study the HJB equation when $\alpha$ goes to zero.

Theorem 3.5 Let the assumptions of Theorem $3.1^{3}$ hold true. If we select

$$
v_{\alpha}(x) \in \operatorname{Arg} \min H\left(x, u_{\alpha}(x)\right)
$$

and

$$
w_{\alpha}=u_{\alpha}-\int_{\mathcal{O}} u_{\alpha}(x) m_{v_{\alpha}}(x) d x
$$

then there exist a constant $\lambda$ and a function $w$ in $W^{2, p}(\mathcal{O})$ such that

$$
\left\{\begin{array}{l}
\alpha w_{\alpha} \rightarrow \lambda \\
w_{\alpha} \rightarrow w \text { weakly in } W^{2, p}(\mathcal{O})
\end{array}\right.
$$

as $\alpha$ goes to zero. The pair $(w, \lambda)$ satisfies

$$
\left\{\begin{array}{l}
L_{0} w+I_{0} w+H(\cdot, w)=\lambda \quad \text { in } \mathcal{O} \\
B w=0 \text { on } \partial \mathcal{O}
\end{array}\right.
$$

for this equation (3.17), the constant solution $\lambda$ is unique.

Proof. We clearly have

$$
\left\|\alpha u_{\alpha}\right\|_{\infty} \leq\|f\|_{\infty} \quad \forall \alpha>0
$$

From the definition of $v_{\alpha}$ we obtain

$$
\left\{\begin{array}{l}
L_{v_{\alpha}} u_{\alpha}+I_{v_{\alpha}} u_{\alpha}+f\left(\cdot, v_{\alpha}\right)=\alpha u_{\alpha} \text { in } \mathcal{O} \\
B u_{\alpha}=0 \text { on } \partial \mathcal{O}
\end{array}\right.
$$

Thus, setting

$$
h_{\alpha}(x)=f\left(x, v_{\alpha}(x)\right)-\alpha u_{\alpha}(x),
$$

we have

$$
L_{v_{\alpha}} w_{\alpha}+I_{v_{\alpha}} w_{\alpha}+h_{\alpha}=0 \text { in } \mathcal{O}
$$

${ }^{3}$ including (1.11) 
and

$$
w_{\alpha}=\int_{0}^{\infty} e^{-\alpha t} \Phi_{v_{\alpha}}(t) h_{\alpha} d t
$$

Since the mean $\bar{h}_{\alpha}$ vanishes, we can repeat the argument in Proposition 3.3 to deduce that

$$
\left\|w_{\alpha}\right\|_{\infty} \leq C, \quad \forall \alpha>0
$$

which implies

$$
\left\|w_{\alpha}\right\|_{W^{2, p}} \leq C, \quad \forall \alpha>0
$$

Hence, by means of (3.18) and (3.22) we obtain

$$
\alpha u_{\alpha}(x) \rightarrow \lambda \text { constant }
$$

using a subsequence converging in $W^{2, p}$ weakly and in $C^{1}(\overline{\mathcal{O}})$ (recall that $p>d$ ) as $\alpha$ go to zero.

Next, conditions (3.22) and (3.23) allows us to take limit as $\alpha$ goes to zero in the nonlinear equation (3.2) to get (3.17).

Finally, under the assumptions of the theorem, there exists a Borel measurable feedback function $\hat{v}=\hat{v}(x)$ such that

$$
\hat{v}(x) \in \operatorname{Arg} \min H(x, w(x)),
$$

and $w$ satisfies

$$
\left\{\begin{array}{l}
L_{\hat{v}} w+I_{\hat{v}} w+f(\cdot, \hat{v})=\lambda \text { in } \mathcal{O}, \\
B w=0 \text { on } \partial \mathcal{O} .
\end{array}\right.
$$

This equation has a unique solution up to an additive constant, according to Proposition 3.3. Notice that the definition of feedback $\hat{v}$ is independent of the constant used to normalize the solution $w$ of equation (3.25).

To show that $\lambda$ is unique, we apply Itö's formula in a convenient way, e.g. Bensoussan [5], in order to get an explicit formula for $\lambda$. Indeed, let $w(x)$ be a solution of the nonlinear equation (3.17), and let $\hat{v}(x)$ be a feedback satisfying (3.24), then $w(x)$ is a solution of 
equation (3.25). Integrating with respect to the invariant measure $m_{\hat{v}}(d x)$ equation (3.25) we obtain

$$
\lambda=\int_{\mathcal{O}} f(x, \hat{v}(x)) m_{\hat{v}}(d x)
$$

Similarly, for any given feedback $v(x)$ we deduce

$$
\lambda \leq \int_{\mathcal{O}} f(x, v(x)) m_{v}(d x),
$$

which completes the proof.

Actually, the above proof also implies the following result.

Corollary 3.6 Under the assumptions of Theorem 3.5 and with the notation of Section 2.1, we deduce that the constant $\lambda$ is the optimal cost, i.e.

$$
\lambda=\inf \left\{J_{x}(v): v(\cdot)\right\}
$$

and the function $\hat{v}$ given by (3.24) is an optimal feedback. Moreover, any solution $w(x)$ of the nonlinear problem (3.17) satisfies

$$
w(x)=\lim _{T \rightarrow \infty} E_{x}^{\hat{v}}\left\{\int_{0}^{T}[f(X(t), \hat{v}(X(t)))-\lambda] d t\right\}+\bar{w},
$$

where $\bar{w}$ denotes the mean of $w(x)$ with respect to the invariant measure $m_{\hat{v}}(d x)$

Proof. It is a classical application Itö's formula, e.g. Bensoussan [5]. Indeed, if $\hat{v}(x)$ is a feedback satisfying (3.24) and $w(x)$ is a solution of equation (3.25) then Itö's formula applied to the function $w$ and the diffusion with jumps $\left(P_{x}^{v}, X(t), t \geq 0\right)$ between 0 and $T$ yields

$$
w(x)=E_{x}^{\hat{v}}\left\{\int_{0}^{T}[f(X(t), \hat{v}(X(t)))-\lambda] d t\right\}+E_{x}^{\hat{v}}\{w(X(T))\} \quad \forall T>0,
$$

which implies (3.27) and

$$
\lambda=\lim _{T \rightarrow \infty}\left\{\frac{1}{T} E_{x}^{\hat{v}} \int_{0}^{T}[f(X(t), \hat{v}(X(t))) d t\} .\right.
$$

On the other hand, if $v(x)$ is any feedback control we have an inequality instead of the equality (3.28), namely

$$
w(x) \leq E_{x}^{v}\left\{\int_{0}^{T}[f(X(t), v(X(t)))-\lambda] d t\right\}+E_{x}^{v}\{w(X(T))\} \quad \forall T>0 .
$$

Hence, we deduce the optimality of $\hat{v}$ and equality (3.26). 
Remark 3.7 Notice that from (3.28) and (3.30) we obtain the representation

$$
w(x)=\inf _{v(\cdot)}\left\{\liminf _{T \rightarrow \infty} E_{x}^{v}\left\{\int_{0}^{T}[f(X(t), v(X(t)))-\lambda] d t\right\}\right\}-\bar{w},
$$

where the infimum is taken over all adapted controls $v(\cdot)$ satisfying

$$
E_{x}^{v}\{w(X(T))\} \rightarrow \bar{w} \text { as } T \rightarrow \infty
$$

This is not a complete characterization of the potential $w(x)$, since we have not established yet the fact that any two solutions of the nonlinear problem (3.17) differs at most in an additive constant. That fact seems to be related to the strictly positivity of the invariant density measure, which in turn is connected with the strong maximum principle. What is possible to establish for any two solutions $w(x)$ and $w^{\prime}(x)$ is the inequality

$$
\left\{\begin{array}{l}
L_{v}\left(w-w^{\prime}\right)+I_{v}\left(w-w^{\prime}\right) \geq 0 \quad \text { in } \mathcal{O}, \\
B\left(w-w^{\prime}\right)=0 \text { on } \partial \mathcal{O},
\end{array}\right.
$$

where $v(x)$ is the feedback associated with the solution $w(x)$. Hence, by means of an argument similar to that of Proposition 3.3 we deduce

$$
w(x)-w^{\prime}(x) \geq \int_{\mathcal{O}}\left[w(x)-w^{\prime}(x)\right] m_{v}(d x) \forall x \text { in } \mathcal{O} .
$$

which implies (cf. Azema et al. [4]) that $w-w^{\prime}$ is a constant expect in a negligible set with respect to the $m_{v}$ measure.

Final Comments: The results obtained in the present paper for ergodic behavior of general diffusions with jumps allow to extend to these processes some of the results in the following papers: (i) Gatarek and Stettner[17], Lions and Perthame [21] and Robin [27] on impulse control, (ii) Menaldi, Robin and Perthame [22] on switching control where only the case of bounded jumps measures was studied, (iii) Robin [29] on perturbation problems in impulse control. By no means these extensions are trivial or useless, on the contrary some careful analysis may be needed to generalize those results. 


\section{REFERENCES}

1 Agmon S, Douglis A, Nirenberg L (1964) Estimates near the boundary for solution of elliptic partial differential equations satisfying general boundary conditions. Comm. Pure Appl. Math. 17:35-92.

2 Anulova SV (1979) On process with Levy generating operators in a half-space. Math. USSR-Izv 13:9-51.

3 Anulova SV (1982) On stochastic differential equations with boundary conditions in a half-space. Math. USSR-Izv 18:423-437.

4 Azema J, Kaplan M, Revu D (1969) Mesure invariante des processus de Markov recurrents. In Seminaire de probabilites III et IV, Lecture Notes in Math. 88, SpringerVerlag, Berlin, 24-33.

5 Bensoussan A (1988) Perturbation methods in optimal control, Wiley, New York.

6 Bensoussan A, Lions JL (1982) Contrôle impulsionnel et inéquations quasi variationnelles, Dunod, Paris.

7 Bony JM (1967) Principe du maximum dans les espaces de Sobolev. Comptes Rendus Acad. Sci. Paris, Serie A 265:333-336.

8 Bony JM, Courrege P, Priouret P (1968) Semi-groupes de Feller sur une variété à bord compacte et problèmes aux limites intégro-differentiels du second ordre donnant lieu au principe du maximum, Ann. Inst. Fourier (Grenoble) 18:369-521.

9 Borkar VS (1991) Topics in Controlled Markov Chains, Pitman Research Notes in Mathematics Series No 240, Longman, Essex.

10 Chaleyat-Maurel M, El Karoui N., Marchal B (1980) Reflexion discontinue et systèmes stochastiques, Ann. Probab. 8:1049-1067.

11 Ethier SN, Kurtz TG (1986) Markov processes, Wiley, New York. 
12 Garroni MG, Menaldi JL (1989) Green's function and invariant density for an integrodifferential operator of second order. Annali di Matematica Pura ed Applicata, Series IV CLIV:117-222.

13 Garroni MG, Menaldi JL (1992) Green functions for second order integral-differential problems, Pitman Research Notes in Mathematics Series No 275, Longman, Essex

14 Garroni MG, Menaldi JL (1995) Maximum principle for integro-differential parabolic operators. Diff. Integral Eq. 8:161-182.

15 Garroni MG, Solonnikov VA (1984) On parabolic oblique derivative problem with Hölder continuous coefficients. Comm. in PDE, 9(14):1323-1372.

16 Garroni MG, Solonnikov VA, Vivaldi MA (1992) Quasilinear Integro-differential parabolic problems with non homogeneous conditions. Houston Journal in Math. 18:481532 .

17 Gatarek D, Stettner L (1990) On the compactness method in general ergodic impulsive control of Markov processes. Stochastics and Stochastic Reports 31:15-25.

18 Gikhman II, Skorokhod AV (1972) Stochastic differential equations, Springer-Verlag, Berlin.

19 Kogan YA (1969) On optimal control of a non-terminating diffusion process with reflection. Theory and Probab. Appl. 14:496-502.

20 Krylov NV (1987) Nonlinear elliptic and parabolic equations of second order, Reidel, Dordrecht.

21 Lions PL, Perthame B (B. 1986) Quasi-variational inequalities and ergodic impulse control. Siam J. Control Optim. 24:512-530.

22 Menaldi JL, Perthame P, Robin M (1990) Ergodic problem for optimal stochastic switching. J. Math. Anal. Appl. 147:512-530.

23 Menaldi JL, Robin M (1984) An ergodic control problem for reflected diffusions with jumps. IMA J. Math. Control Inform. 1:309-322. 
24 Menaldi JL, Robin M (1985) Reflected diffusion processes with jumps. Ann. Probab. 13:319-341.

25 Menaldi JL, Robin M (1989) On the asymptotic behavior of stopping time problems. Boll. Unione Mat. Ital., Serie 7 3-B:723-734.

26 Morimoto H (1991) On average cost stopping time problems. Probab. Theory Related Fields 90:469-490.

27 Robin M (1981) On some impulse control problems with long-run average cost. Siam J. Control Optim. 19:333-358.

28 Robin M (1983) Long-term average cost control problems for continuous time Markov processes: A survey. Acta Appl. Math. 1:281-299.

29 Robin M (1986) On some perturbation problems in optimal stopping and impulse control. In Stochastic Differential Systems, Stochastic Control Theory and Applications, Eds.: W. Fleming and P.L. Lions, IMA Vol. 10, Springer-Verlag, New York, 473-500.

30 Stettner L (1989) On ergodic stopping and impulsive control problems for nonuniformly ergodic Markov processes. Appl. Math. Optim. 19:75-95. 